\title{
LXXXI. On the colours of mixed plates-Part II
}

\section{C.V. Raman M.A. \& Bhabonath Banerji M.Sc.}

To cite this article: C.V. Raman M.A. \& Bhabonath Banerji M.Sc. (1921) LXXXI. On the colours of mixed plates_Part II, Philosophical Magazine Series 6, 41:246, 860-871, DOI: $10.1080 / 14786442108636280$

To link to this article: http://dx.doi.org/10.1080/14786442108636280

曲 Published online: 08 Apr 2009.

Submit your article to this journal

Џ Article views: 4

Q View related articles 두 
860 Profs. C. V. Raman and Bhabonath Banerji on

and the products $\mu y z \mathrm{~S} y, \mu z x \mathrm{~S} x$, and $\mu x y \mathrm{~S} x \mathrm{~S} y$ are plotted against the dry densities. It will be noted that the points. involving $\mu x y$ lie sensibly on a straight line, whilst those involving $\mu y z$ and $\mu z x$ lie approximately on two parallel straight lines.

Relations similar to the above have been obtained for each of three values of Young's Modulus and six values of Poisson's Ratio for spruce, which the writer hopes shortly to publish, and these were found to agree with the symmetrical ratios of a body having three planes of elastic symmetry *. It may be noted that the number of annual rings per inch is not involved in any of the relations.

The writer wishes to thank the late Principal, J. C. M Garnett, M.A., for providing facilities for conducting the research, and also Professor G. Stoney, F.R.S., for many helpful suggestions. He is further much indebted to Mr. A. L. McAulay, B.Sc.Tas., for valuable assistance with the experiments.

LXXXI. On the Colours of Mixed Plates.-Part II. By C. V. Raman, M.A., Palit Professor of Physics, and Bhabonath BanerJi, M.Sc., Assistant Professor of Physics, University of Calcutta $\uparrow$.

1. Inadequacy of the Elementary Diffraction Theory.

THE optical phenomena exhibited by mixed plates have been described in detail, with illustrations, in the first part of the paper. We now proceed to consider their explanation. It is obvious that the phenomena must be classed as laminar diffraction effects. But, as has already been remarked in the introduction to the first part of the paper, many of the features observed differ from what one might expect on the usual elementary theory of diffraction phenomena. Conspicuous amongst these is the special character of the diffraction-halo seen surrounding a distant light-source viewed through a mixed plate of uniform thickness. Many of the observed features of this halo are not explicable on the elementary diffraction theory : namely, the succession of dark and bright rings of widths rapidiy increasing from the centre outwards, the perfect blackness of

* Love, 'The Mathematical Theory of Elasticity' Art. 73.

$\uparrow$ Communicated by the Authors. 
the dark rings in the outer part of the halo, and the obviously composite structure of the inner part of the halo. In seeking for an explanation of these effects, a clue is furnished by the observation already recorded, that when a mixed plate is observed by the light diffracted by it, the whole surface of the film does not appear luminous, but only the laminar boundaries or lines of separation of the two media forming the film. The optical effects of mixed plates are thus, in fact, the optical effects due to the scattering or radiation of light from laminar diffracting boundaries. It is necessary to stndy the manner in which an individual laminar boundary scattors or diffracts light incident on it ; to compare this with the indications of theory, and from the observed effects to infer the aggregate result of the scattering by a large number of such boundaries irregularly situated on the film. These points we now proceed to discuss.

\section{Examination of Mixed Plates by the Method of the Foucault Test.}

The most convenient way of examining the scattering of light by a laminar boundary in directions nearly coincident with that of the incident waves is by the method of observation known as the Foucault knife-edge test or the Töpler Schlieren method. The theory of this method was developed by the late Lord Rayleigh on the basis of the usual elementary treatment of diffraction phenomena ${ }^{*}$, and it was shown by him that a discontinuous laminar boundary should appear as a luminous line when examined by the method of the Foucault test. A beautiful illustration of Rayleigh's theory is furnished on examining a clear piece of mica by the Foucault test, when it will be found that the striæ or boundaries in the mica between regions having slightly different thicknesses shine out as vividly-coloured lines of light in a dark field + . To study the phenomena of mixed plates by a similar method, the following arrangement is suitable. Light from a small circular aperture illuminated by an incandescent filament lamp falls upon a good achromatic lens, and is brought to a focus at a distance from it. Two

* "On Methods for Detecting Small Optical Retardations and the Theory of the Foucault Test." Phil. Mag. Feb. 1917.

+ "On the Colours of the Striæ in Nica," C. V. Raman and P. N. Ghosh. 'Nature,' October 1918 See also P. N. Ghosh, Proc. Roy. Soc. A. Series, vol. xcvi. p. 257 (1919).

Phil. Mag. S. 6. Vol. 41. No. 246. June $1921 \quad 3 \mathrm{~L}$ 


\section{Profs. (. V. Raman and Bhabonath Banerji on}

pieces of good plate-glass, pressed together with a drop or two of water mixed with air between them, are placed in front of the achromatic lens. The film thus enclosed between the glasses forms the "mixed plate," which is observed through a telescope placed with its objective just behind the focus of the achromatic lens. The appearance of the film, as seen with this arrangement, depends on the form of the aperture or stop regulating the admission of light from the focal plane of the lens into the object-glass of the observing-telescope:-(A) With an aperture placed centrally in the focal plane so as to admit the light coming to the geometrical focus but cutting off the diffracted light, the water-air boundaries in the mixed plate appear as coloured lines in a bright white field. (B) When a central stop is placed symmetrically so as to cut off the light coming geometrically to a focus, and an annular aperture surrounding the stop admits only diffracted light into the observing telescope, the whole field appears dark, except the water-air boundaries, which are seen apparently doubled, shining out as two brightly-coloured lines of light running parallel to each other and separated by a fine perfectly dark line coinciding with the exact outline of the boundary. The colours seen depend only on the thickness of the film, and are independent of the size or shape of the boundaries. They are complementary to those seen in case A. A magnified photograph of the film under these conditions, showing the apparent doubling of the boundaries, was reproduced in fig. 4 of the Plate accompanying the first part of the paper. (C) When, instead of a symmetrical annular aperture, we have only a small aperture placed eccentrically in the focal plane admitting diffracted light into the observing telescope, then the full outlines of the water-air boundaries are not seen, but only two small portions of each closed boundary, such that the normals to the boundary at the two points visible are parallel to the radius vector joining the focus with the aperture placed in the focal plane. With such an excentrically-placed aperture the phenomenon of the doubling of the boundaries noticed under (B) does not occnr, and we merely get a single luminous coloured line in a dark field running along the portions of the boundary visible.

The phenomena described above are closely analogous to those exhibited by the striæ in mica*. The observations show clearly that the laminar boundaries in a mixed plate

* P. N. Ghosh, loc. cit. 
sct as centres or sources of diffracted radiation. Each element of a laminar boundary may be regarded as sending out tuo streams of radiation-one on the more retarded, and one on the less retarded side of the wave-front. In directions nearly coincident with that of regular transmission of the incident waves, these two streams are of practically equal intensity and of opposite phases. In such directions the phenomena observed are in agreement with the indications of Lord Rayleigh's theory, according to whish the colour of the laminar boundary, as seen in the Foncault test, should be complementary to the colour of the central fringe in the laminar diffraction pattern produced by it. For very small angles of diffraction, therefore, the elementary diffraction theory gives results which are substantially valid. As we shall see presently, this ceases to be true when we consider larger angles of diffraction.

\section{The Unsymmetrical Scattering of Light by Laminar Boundaries: Normal Incidence.}

Very simple observation suffices to show that the scattering of light through larger angles by laminar boundaries exhibits features not indicated by the elementary theory. For this purpose, a thin film of liquid mixed with air enclosed between two glass plates is placed normally in the track of a strong pencil of light from a lantern, and viewed obliquely by the eye with or without the aid of a magnifier. It will be noticed at once that the edges of separation of liquid and air diffract light in a strikingly unsymmetrical manner. Any given edge can easily be observed diffracting light at all angles np to $90^{\circ}$ when viewed on the side passing through the liquid; but viewed on the side passing through air, it can hardly be seen at all except in directions making less than about $10^{\circ}$ or $15^{\circ}$ with the direction of the incident beam, so small is the intensity of the diffracted light in this region. If a closed curved boundary enclosing air be viewed at a slight obliquity to the direction of the incident light, the two limited portions of the boundary visible appear differently coloured, one being much fainter than the other. The fainter portion which is seen through air vanishes altogether when viewed at greater obliquity, while the part of the boundary seen through the liquid, $i . e$. on the more retarded side of the wave-front, remains visible throughout, its colour changing periodically and becoming richer as the obliquity of observation is increased, $3 \mathrm{~L} 2$ 


\section{Profs. C. V. Raman and Bhabonath Banerji on}

and finally appearing achromatic when viewed in a direction nearly parallel to the plate. An edge too thick to show colour when observed in a direction nearly normal to the plate, will appear vividly coloured when viewed obliquely on one side of this direction, and be practically invisible from the other side. It should also be remarked that in oblique directions the portions of any one curved boundary that can be seen at a time become greatly reduced-in fact, nearly contract to single points. The normal to the boundary at these points lies in the plane of observation. Each element of a curved boundary is therefore effective in scattering light principally in a plane normal to its own direction *.

For a closer examination of the manner in which the colour of the light scattered by a laminar boundary varies with the direction of observation, the mixed plate may be placed on the table of a spectrometer, and viewed through a low-power microscope which replaces the telescope ordinarily used in the instrument. The laminar boundaries under observation should be illuminated by a somewhat narrow pencil normally incident on the plate, and in order to screen this from entering directly into the field of view of the microscope, a wire may be plaoed immediately in front of the objective. On turning the microscope about the axis of the spectrometer, the phenomena described in the preceding paragraph may be readily observed and studied. Viewed nearly in the direction of the incident light, the laminar boundaries appear of a uniform colour depending on the thickness of the film. On turning the microscope aside to a slightly oblique direction, each of the boundaries seen changes colour, but differently in its two parts which are seen respectively through the more and less refrangible media. For instance, a closed houndary which, seen in a nearly normal direction, appears throughout golden yellow, viewed at a slightly greater obliquity, appears red on the portion seen through the liquid and greenish blue on the portion seen through air, the latter appearing much fainter. The colours and intensities of the two parts of each boundary are interchanged when the microscope is turned over to the other side. Viewed at still greater obliquities, further fluctuations of colour occur, the sequence of these variations being quite asymmetric with respect to the two sides of the direction of the regularly transmitted pencil. A clear idea

* This is generally true of all curved diffracting boundaries on which light is normally incident. See Phil. Mag. Jan. 1919, p. 127, and Sept. 1919, p. 219. 
of these phenomena will be obtained from the diagram given below.

Fig. 1.

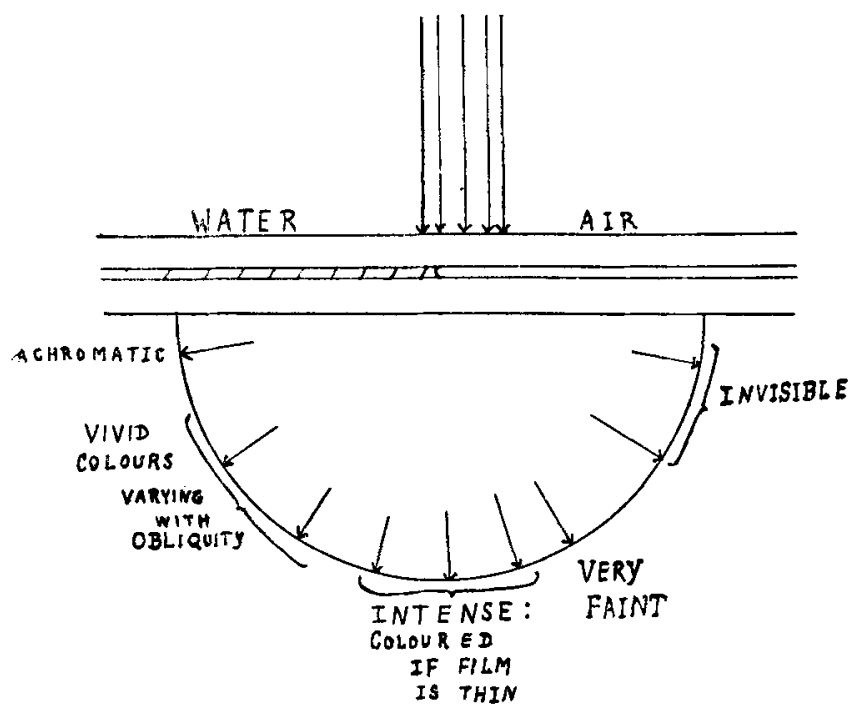

The fluctuations of colour with obliquity are most striking in the region on the left of the diagram where they commence, and are continued over to the right, where they are much less vivid, apart from the greatly decreased intensity of the diffracted light on this side. If, instead of white light, a pencil of monochromatic light be used to illuminate a laminar boundary, the light scattered by it shows a similar asymmetry, the intensity fluctuating as the obliquity is varied, and vanishing in a series of directions which are closer together or wider apart according to the thickness of the film.

\section{Explanation of the Diffraction-Haloes.}

We are now in a position, on the basis of the observations described in the preceding section, to form a general idea of the manner in which the diffraction-haloes due to a mixed plate arise. To begin with, it may be assumed that the mixed plate is of uniform thickness, and that the light is incident normally upon it. Consider the light diffracted by the film in a direction making an angle $\theta$ with the incident pencil of rays. To the aggregate scattering in 


\section{Profs. C. V. Raman and Bhabonath Banerji on}

this direction the laminar boundaries in the film all contribute, the effective elements of each boundary being those normal to the plane containing the incident and scattered rays. Since, as we have seen, each boundary scatters light in an insymmetrical manner with respect to the direction of the transmitted pencil, we may divide all the effective elements into two groups, namely those which scatter light in the given direction respectively through the more and less refrangible media in the film. In summing up the effects of the elements in each group in the given direction $\theta$, the relative phases of the scattered rays which depend on the positions of the scattering elements have to be taken into account; and as the boundaries are irregularly distributed on the film, the phases of the elements in each group may be assumed to valy arbitrarily. Further, as the dimension of each boundary - that is, the diameter of each air-bubble in the film-varies between wide limits, there is also no fixed phase-relation between the corresponding elements in the two groups. Thus, the intensity of the scattered light in the direction $\theta$ is statistically equal to the sum of the intensities due to the two groups separately, and the intensity due to each group is, similarly, the sum of the intensities due to its discrete elements. But for individual values of $\theta$ there may be large deviations from the statistical average, and this gives rise to the granular structure of the halo in monochromatic light, and its radial fibrous structure in white light * $W_{\theta}$ have seen that the colour and intensity of the scattering by each elementary boundary varies with $\theta$ in a manner depending on the thickness of the film, and since this is constant, the average aggregate effect of each group varies. with $\theta$ in the same way as for individual elements. Considering together all the possible directions of the scattered pencil, we see that each group will give rise to a diffractionhalo with circular rings surrounding the direction of the source. The diffraction-halo due to one group extends from $\theta=0^{\circ}$ up to $\theta=90^{\circ}$, while that due to the other group is of sensible intensity only for small values of $\theta$. Near the centre, therefore, the two sets of rings due to both groups of elements are superposed and the halo is composite; while in the outer part, only those elements which diffract light through the more refrangible medium have a sensible effect, and the halo is therefore simple. It is thus seen that

* Compare De Hås, "On the Scattering of Light by Small Particles." Proc. Roy. Soc. of Amsterdam, 1918 p. 1278; also Lord Rayleigh, Phil. Mag. Dec. 1918. 
the summation of the effects of the individual boundaries in the film leads to results in close agreement with the features of the halo already described.

The foregoing treatment also enables us at once to explain the increased intensity of the halo in certain directions in the case of films containing elongated boundaries. In so far as relates to the general configuration of the halo, the arguments of the preceding paragraph apply mutatis mutandis also in the case of such films, and it is clear why the distortion of the boundaries leaves the circular form and positions of the rings in the diffraction-halo unaffected; the only difference is as regards the relative intensity of the halo along different radii, which depends on the aggregate length of the scattering elements effective along the respective directions. If we divide up each boundary in the film into $n$ parts, such that the successive normals at the points of division make angles of $2 \pi / n$ with each other, the aggregate scattering effect of each of the $n$ groups of parallel elements in the film in the plines respectively normal to them would be the same, provided the average length of an element in each of the $n$ groups were the same. The latter condition is satisfied, provided the boundaries in the film show no bias towards elongation in any particular direction. But if they do show such bias, the average length of an element is greatest in respect of the groups running parallel to the general direction of elongation. and least in the groups running transverse to such direction. From this, it follows that the intensity of the halo should be greatest in the plane perpendicular to the direction of elongation, and least in the plane parallel to it. This is exactly what is observed. The intensities should, in fact, be quantitatively proportional to the square of the average length of an element in each group.

\section{Mathematical Theory: Normal Incidence.}

We have now to consider the explanation of the unsymmetrical scattering by the laminar boundaries in a mixed plate, and to express the results in quantitative form. No rigorous treatment of the problem of diffraction by plane transparent lamina bounded by edges appears as yet to have been put forward. In practice, the precise shape of the diffracting-edge should obviously have a considerable influence in determining the manner in which it scatters light in directions much removed from that of regular propagation of the incident waves. For instance, the striæ in mica are 


\section{Profs. C. V. Raman and Bhabonath Banerji on}

often found, under the microscope, to possess an echelonlike structure*. The striæ diffract light asymmetrically through large angles, but the effects observed with different striæ differ in a manner which suggests that the results are influenced by the structure of the laminar edge as well as by its total thickness. In the case of mixed plates, owing to the action of surface tension, the laminar edges are not perpendicular to the surface of the film, but have the form of a moniscus (fig. 2). We shall assume that the angle of contact of the liquid with the plate is zero, and that the meniscus is of semicircular form. The diagram is assumed to be drawn perpendicular to the plane of the film and also to the element of the scatteriug boundary under consideration.

Fig. 2.

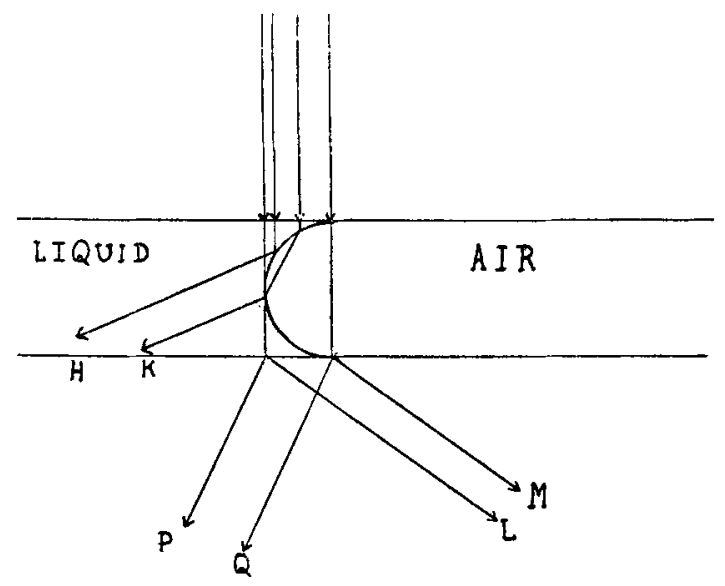

It is obvious, especially in view of the curvature of the surface of separation, that the incident light would be scattered very differently towards the two sides of the boundary between the two media. On either side, scattered disturbances would emerge which have traversed different paths partly through one medium and partly through the other, and the problem is to find their relative intensities and the path-differences under which they interfere. As regards the light scattered towards the side of the nore refrangible medium, it is convenient to assume tentatively that the paths traversed are those given by the laws of

* P. N. Ghosh, "On some Phenomena of Laminar Diffraction observed with Mica." Proc. Ind. Assoc. for the Cultivation of Scien ce Galcutta, vol. vi. pt. i. (1920). 
geometrical optics. Part of the light incident on the curved meniscus would be reflected within the liquid, and if the angle of incidence were greater than the critical angle, the reflexion would be total. Part would also be refracted into the rarer medium, and after a second refraction emerge again into the liquid. If these disturbances finally travel in parallel directions, such as those shown by $\mathrm{H}$ and $\mathrm{K}$ in the plane of the diagram, the path-difference between them may be readily evaluated and shown to be

$$
t(1-\mu \sin i)\left(\mu \cos i-\sqrt{1-\mu^{2} \sin ^{2} i}\right)+\delta \text {. . . }
$$

In this formula, $t$ is the thickness of the film, $\mu$ the refractive index of the liquid, and $i$ the angle of incidence on the meniscus of the light which is twice refracted. $\delta$ is the correction necessary on account of the change of phase in total reflexion. If $i$ be nearly equal to zero, both pencils emerge nearly parallel to the direction of the incident rays, $\delta$ has the value which obtains at nearly grazing incidence, and the expression for the path-difference reduces to

$$
(\mu-1) t-\lambda / 2 \text {. . . . . . . }
$$

The light scattered through small angles thus interferes under a difference of path which is the same as that of the segularly-transmitted pencils less half a wave-length. 'This agrees with what we should expect on the simple diffraction theory, the scattered light being of a colour complementary to that due to the interference of the regularly-transmitted pencils. For larger angles of scattering, the difference of path between the interfering pencils given by (1) steadily falls off in magnitude, and finally becomes zero when the angle of incidence on the meniscus is just equal to the eritical angle, as the pencils then become coincident and $\delta$ is also equal to zero. We should thrs expect to observe a series of maxima and minima of intensity in the scattered light in different directions, which is exactly what is found in experiment. The deviation of the interfering pencils within the liquid film is given by $2(r-i)$ where $\mu \sin i=\sin r$, and when this is equal to $(\pi-2 \alpha)$ where $\alpha$ is the critical angle, the path-difference vanishes, and we should expect the scattered light to be achromatic. The angle of scattering $\theta$ on emergence from the film is given by the relation $\sin \theta=\mu \sin 2(r-i)$. It is worthy of note that, as the correction $\delta$ for the change of phase in total reflexion depends on the plane of polarization of the incident light, the positions of the maxima and minima in the scattered light should be slightly different for light polarized in and at right angles 
to the plane of incidence, the difference being greatest wher the path-difference given by (1) is rather small. This point. will be noticed again hereafter.

We should, of course, also consider disturbances such as those indicated by $\mathrm{P}$ and $\mathrm{Q}$ in. the diagram, which have traversed paths lying wholly in one medium or the other and are then diffracted in oblique directions from the edges of the regularly-transmitted wave-fronts. If $\theta$ be the angle of diffraction on emergence from the film, the path-difference under which such disturbances interfere is

$$
(\mu-1) t-t / 2 . \sin \theta-\lambda / 2 \text {. . }
$$

the deduction of $\lambda / 2$ being made on account of the phasereversal of the diffracted ray. When the angle of seattering is not large, the path-differences given by equations (3) and (1) are identical, as may be readily shown on expanding (1) and neglecting the second and higher powers of $i$. Indeed, in this case it follows from the well-known principle of minimum or stationary path, that the actual course followed within the film by either of the interfering disturbances is a matter of indifference, provided the deviations from the geometrical path are not large. On the other band, when the angle of scattering is large, the intensity of the light scattered from the curved interface between the two media towards the more refrangible medium would be far larger than that diffracted from the edges of the wave-fronts. Hence, both for small and large angles of scattering we wonld be justitied in regarding the expression (1) for the path-difference as substantially valid.

Passing on now to consider the light scattered towards the less refrangible medium, it is clear in this case that no sensible portion of it is contributed by the curved interface between the media. The scattered light which emerges consists entirely of disturbances (such as those indicated by $I_{2}$ and $M$ in the diagram) diffracted from the edges of the wave-fronts. These interfere under a path-difference

$$
(\mu-1) t+t / 2 . \sin \theta+\lambda / 2, \quad . \quad . \quad . \quad \text {. }
$$

which now increases with the increasing obliquity of the diffracted light; in directions nearly normal to the film the scattered light is, as before, of a colour complementary to that dne to the interference of the regularly-transmitted pencils, and its intensity is equal or comparable with that of the light similarly diffracted towards the side of the more refrangible medium. At larger angles of diffraction, however, the intensity falls off with great rapidity, and is far less than on the side of the more refrangible medium where the light scattered from the curved interface plays an important part. 
We have not, so far, discussed the relative intensity of the interfering pencils scattered in any given direction. The mathematical treatment of this question may be deferred till a later stage. For the present, it may suffice to remark that experimental observation as already detailed shows the interferences to be remarkably perfect, and hence the interfering pencils must be of comparable or equal intensity throughout the region in which we have maxima and minima in the scattered light. There is no difficulty in understand. ing, at least in a general way, why this is the case. In directions nearly normal to the film, light is diffracted chiefly from the wave-fronts regularly transmitted through the film, and the contributions to the scattered radiation from the part of the wave-front lying on either side of each boundary should obviously be equal. In more oblique directions the scattering occurs chiefly at the curved interface between the two media, and a calculation on the principles of geometrical optics shows the intensities of the pencils emerging respectively after two refractions and after total reflexion to be comparable throughout, the intensity of the former being at first greater, then equal and finally less than that of the latter, as the angle of scattering increases. Thus there is reason to expect that throughout the range in which the scattered light can be observed, the interferences should be strongly marked.

\section{Concluding Romarks.}

Numerical computation of the position of the dark and bright rings in the halo from formula (1) of the preceding section gives results in general agreement with experiment. The width of the successive rings increases rapidly as we proceed outwards from the centre of the halo. For instance, in the particular case of a film for which the pathdifference for normal transmission through the two media is five wave-lengths, the angular radii of the five dark rings in the halo as given by the formula are $\theta=0^{\circ}, \theta=9^{\circ} 20^{\prime}$, $\theta=20^{\circ} 27^{\prime}, \theta=37^{\circ} 12^{\prime}$, and $\theta=73^{\circ}$, respectively. These quantities are of the same order as those actually observed.

A detailed quantitative comparison between experiment and theory, together with a discussion of the theory of the diffraction-haloes due to obliquely-held plates and of the effects observed with non-uniform plates, as also of the special phenomena observed with dry films of albumen, will be given in the concluding instalments of the paper.

Caleutta, India,

Dated the 14th of October, 1920. 